\title{
The Cyberith - Virtualizer
}

This device developed by the young virtual-reality-enthusiasts of "Cyberith" is a locomotion device that delivers completely new impressions and experiences regarding gameplay and entertainment.

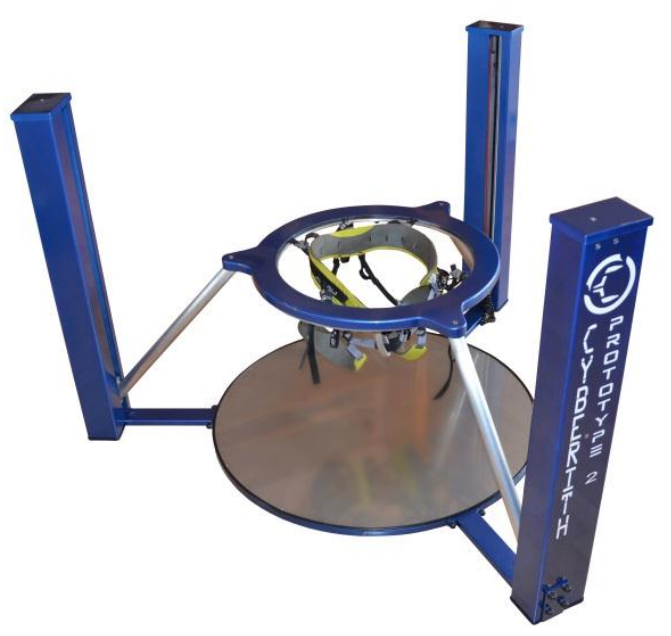

Realistic experiences in your own private home It delivers the most realistic experience of virtual worlds possible - in your own private home. The Virtualizer was developed with the goal in mind to optimize the realistic impression of first-person games but free the player from his chair and place him right into the action. Using the Virtualizer yields the opportunity to actively participate in the adventures of your favourite character and for the first time not to be stopped by the borders of your screen.

\section{Wide range of natural movements}

This is possible because the device is not hindering your natural freedom of movement. The Virtualizer supports not only simple forward-faced movements like walking or running, but also walking backwards, crouching, jumping and even sitting. The device is world's first omnidirectional treadmill for home use that has its sensor system integrated. The highly engineered sensor system combined with VR-glasses that support 3D places the user directly into the virtual world and delivers overwhelming experiences.

\section{Stepless movements without the need of special shoes}

These experiences are enhanced by the Virtualizer's ability to detect and process movement stepless which makes it possible to move in the same speed ingame as you do in the real world. It was a high matter of interest for the developers to make the device usable without the need for special shoes that would only imply additional costs for the customer and problems regarding shoe sizes. The underlying functional principle is based on a special platform that features a highly elaborated surface with the right coefficient of friction and a vertically adjustable ring-construction in hip-height that absorbs the remaining frictional force. The ring was designed to enable the user free movement and is adjustable to the operators specific

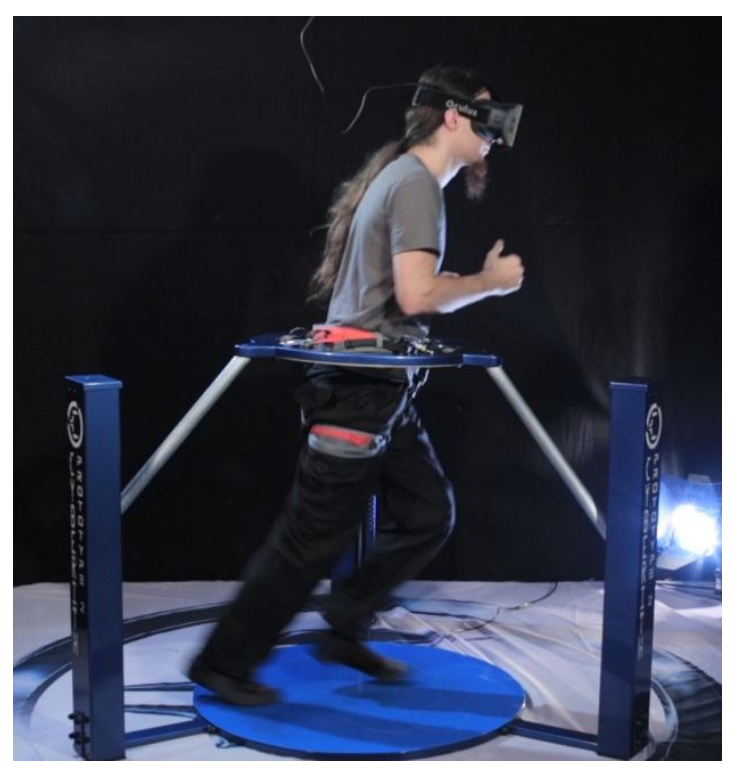
body height. 


\title{
Easy to install and whisper quiet
}

Additionally, the device is designed to work with "plug and play" and thus it is very easy to set up and install. Because the Virtualizer is used with plain socks it does not yield the risk of noise disturbance.

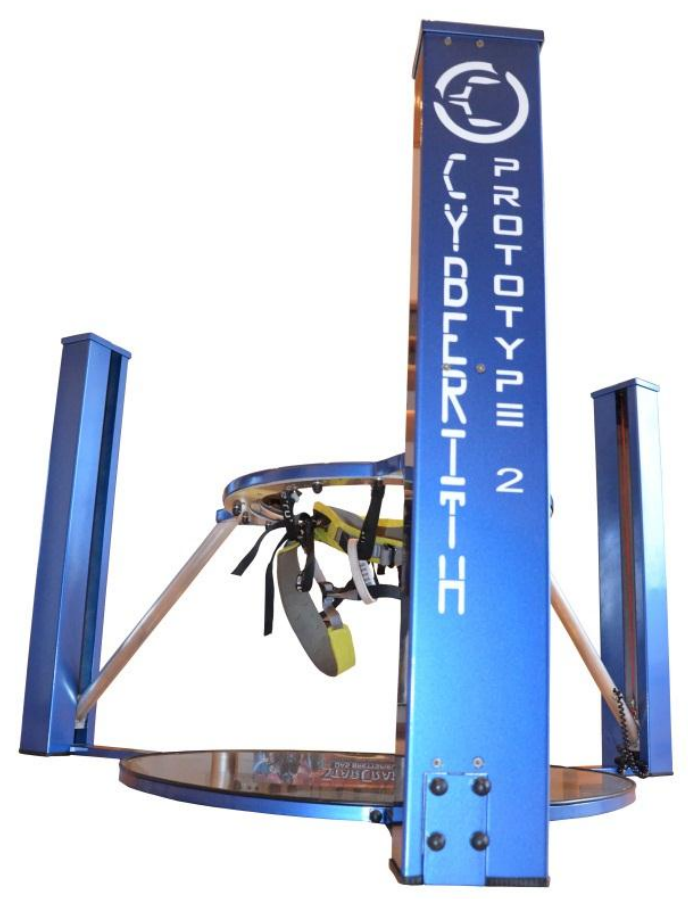

\begin{abstract}
All-purpose Device
The fields of application of the Virtualizer go much further than just gaming. Because the device enables joining any virtual world you can imagine, there are a lot of possible applications in fields like architecture, tourism, psychological therapy, Education, Training,...
\end{abstract}

If you desire more information or you have questions regarding the device, visit our homepage at www.cyberith.com or write us to info@cyberith.com.

\section{Cyberith online:}

www.cyberith.com

www.facebook.com/cyberith

www.youtube.com/cyberith

www.twitter.com/cyberith

www.instagram.com/cyberith\#

\section{Press Contact:}

Tuncay Cakmak

info@cyberith.com

+43 (0) 6506363260

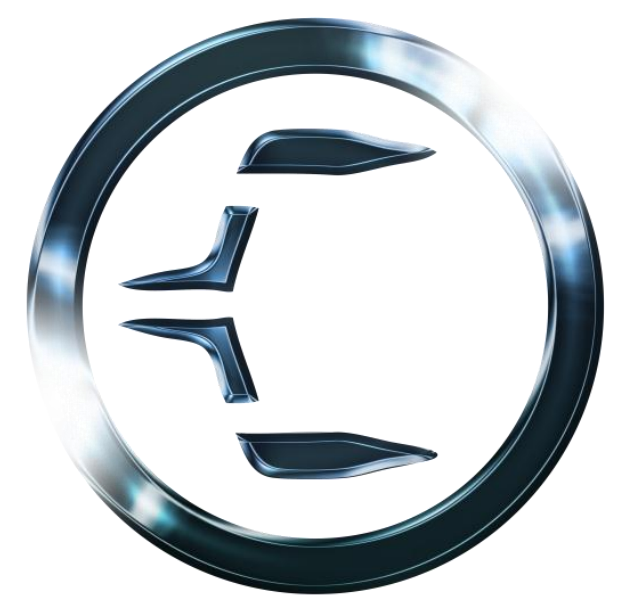

